\title{
Modo de vida rural e urbano: a criação de índices vinculados à sociedade de mercado
}

\section{Rural and urban way of life: an index established in the market society}

\section{Gustavo Bastos Braga}

gustavo.braga@ufv.br

Doutor em Extensão Rural. Professor do Departamento de Economia Rural na

Universidade Federal de Viçosa (UFV)

Ana Louise de Carvalho Fiuza

louisefiuza@ufv.br

Doutorado em Ciências Agrícolas pela Universidade Federal Rural do Rio de Janeiro (UFRRJ). Professora do Departamento de Economia Rural na Universidade Federal de Viçosa (UFV)

\section{Roberto Max Profil}

roberto.protil@ufv.br

Doutor em Ciências Econômica pela Georg-august-universität. Professor do

Departamento de Economia Rural na Universidade Federal de Viçosa (UFV) 


\title{
RESUMO
}

A discussão acerca das transformações do modo vida em sociedades rurais ancora-se, inicialmente, na própria delimitação entre o que deve ser considerado rural e o que deve ser considerado urbano. Norteando-se pela ótica de autores como Abramovay, o presente artigo tem como objetivo debater sobre a criação de um "índice de urbanidade e ruralidade". Tal índice poderá ser empregado para demonstrar a modificação do modo de vida rural. Utilizando a metodologia Analytic Hierarchy Process aplicada em estudantes de mestrado e doutorado em Extensão Rural da Universidade Federal de Viçosa procurou-se estabelecer os vetores para a criação de tal índice. Os resultados apontaram para uma consonância entre o uso de variáveis ligadas ao modo de vida nessa definição.

Palavras-chave: Modo de Vida. Urbano. Rural.

\begin{abstract}
The discussion about the changes in the rural lifestyle is, initially, pass by define what is rural and what should be considered urban. Guided by authors such as Abramovay, this article aims at discussing the creation of an "urbanity and rurality index". This index should be employed to demonstrate the ways of transformations in the rural lifestyle. This paper uses the Analytic Hierarchy Process methodology. The research universe is composed by master and doctorate students of Rural Extension postgraduate program from the Universidade Federal de Viçosa (Brazil). We attempt establish vectors for creation of a rurality index. The results indicated a harmony for the usage of variables connected with the lifestyle by this definition.
\end{abstract}

Keywords: Lifestyle. Urban. Rural. 


\section{Introdução}

Os dados do Instituto Brasileiro de Geografia e Estatística (IBGE) mostram que, seguindo a adoção de rural e urbano dada pelo Estado, o Brasil, oficialmente, tem um crescimento em sua população urbana. O índice chamado de Grau de Urbanização mensurado pelo IBGE mostra, em 1991, um Brasil com 75,59\% da população urbanizada e, em 2006, esse número subiu para 84,53\%. No entanto, sob a perspectiva de vários autores, como Veiga (2002), o Brasil é mais urbano do que apontam os números oficiais apresentados.

Veiga (2002) chama a atenção para a importância de se diferenciar os tipos de cidades existentes no território brasileiro, tais como: "cidades rurais", "centros urbanos" e "megalópoles", as quais seriam tratadas genericamente como cidades, dificultando o direcionamento racional das políticas públicas a elas endereçadas. Este autor tem recebido muitas críticas de geógrafos e cientistas sociais que argumentam que os indicadores por ele utilizados para caracterizar o espaço, tais como a densidade demográfica, o tamanho da população e a distância dos centros urbanos, se centraram em aspectos físicos e desconsideravam aqueles referentes aos "modos de vida" e mesmo aos "meios de vida". Para Sanches-Casas (2009), o espaço deveria ser percebido como a junção do modo de assentamento, identificando a estrutura do meio físico, tanto em termos da sua formação social construída mediante a sua produção e uso, assim como em termos das características ecológicas-ambientais.

No entanto, mesmo na corrente que toma os modos ou os meios de vida na definição de urbano e rural há diversas divergências sobre quais pesos e proxies devem ser utilizados na mensuração desta definição. Uma das formas de realizar esse estudo é a estruturação de um índice de integração à sociedade capitalista de mercado.

Assim, o presente estudo tem como escopo estruturar e dar pesos aos proxies através do uso da metodologia Analytic Hierarchy Process (AHP) para estruturar a importância das variáveis para a criação do índice proposto. 


\section{Referencial teórico: Um breve apanhado do estado da arte da definição de urbano/rural}

Segundo Kageyama (2008), historicamente, a distinção entre rural e urbano foi realizada pelo critério ocupacional. O rural era considerado como um ambiente de predominância natural onde se realizavam atividades agrícolas. Neste espaço a densidade populacional seria relativamente pequena, com menor possibilidade de mobilidade social e o menor número de contatos. Na literatura, podemos encontrar várias formas em uso para a delimitação entre o urbano e o rural, dentre elas, a que está ligada ao patamar demográfico de uma região. Segundo Endlich (2010), essa concepção define o rural como dispersão e o urbano como aglomeração.

Esse critério é bastante difundido, até por sua facilidade, em países como Itália, Grécia, Argentina e Panamá (ABRAMOVAY, 2000). Uma das dificuldades dessa forma de delimitação é encontrar o patamar de corte que varia de acordo com a nação: enquanto na Noruega é adotado o número de 200 habitantes, no Japão esse número é de 50.000 habitantes (BEAUJEU-GARNIER, 1997). Rybczynski (1996) é um crítico dessa forma de separação entre urbano e rural. Para o autor, essa é uma avaliação rude para aferir urbanidade e ruralidade, dado que essa forma não vislumbra a riqueza dos habitantes, bem como sua educação e cultura. Para Abramovay (2000), esse critério não permite a abordagem regional da ruralidade, uma vez que classifica determinado local como urbano ou rural, contudo impossibilita indicar se determinada região é mais ou menos rural. Essa forma de distinção entre campo e cidade, delimitada pela concentração regional, não seria muito utilizada pela dificuldade na demarcação precisa dos limites da área urbana, e também possui as mesmas desvantagens do método que considera o patamar demográfico supracitado (BERNARDELLI, 2010).

A ocupação econômica da população é também considerada uma forma de limitação entre rural e urbano. Países como Israel adotam essa forma, onde é considerada urbana a localidade na qual pelo menos dois terços dos chefes de família exercem atividades não agrícolas (ABRAMOVAY, 2000). Esse ponto de vista congrega o rural às atividades de natureza primária, contudo esse método também tem suas limitações. As atividades não-agrícolas vêm crescendo no meio rural, sem mencionar que a adoção desse procedimento baniria os espaços rurais dos países desenvolvidos (ENDLICH, 2010). Outra delimitação utilizada é a delimitação administrativa - forma utilizada no Brasil. 
Aqui, a noção de diferenciação entre rural e urbano ocorre de forma arbitrária pelo poder público.

Abramovay (2000, p. 37) crítica em três pontos essa forma de divisão:

- O rural é definido, ao menos em parte, pelo arbítrio dos poderes públicos municipais e as consequências fiscais da definição acabam sendo mais importantes que seus aspectos geográficos, sociais, econômicos ou culturais;

- Desde que haja extensão de serviços públicos a certo aglomerado populacional, ele tenderá a ser definido como urbano: é assim que, no Brasil, as sedes de distrito com algumas centenas ou dezenas de casas são definidas como "urbanas";

- O rural tenderá a ser definido, em princípio, pela carência, o que não pode ser considerado um critério adequado sob qualquer ponto de vista.

Dada a quantidade de desvantagens dessa forma de delimitação, é crescente o descontentamento com essa metodologia de definição do rural. Assim, se apresenta demasiado complexa a definição entre urbano e rural. São perceptíveis as limitações de formas pragmáticas e descontextualizadas na tentativa de limitar o urbano e o rural. No caso brasileiro, Braga et al. (2016) demonstram que a utilização de definições internacionais de ruralidade levantam as distorções em relação às definições oficiais. Essas visões tradicionais do rural demonstram-se ultrapassadas e estimativas, como o "grau de urbanização" do IBGE, estão obsoletas (BERNARDELLI, 2010). Buscando a quebra dos paradigmas, no sentido kuhniano da palavra, surge a visão de um novo rural.

\section{Analytic Hierarchy Process - AHP}

Os métodos de apoio ou auxílio multicritério à decisão surgem na década de 1970 com o escopo de resolver dificuldades, em situações específicas, onde o tomador de decisão deveria solucionar um problema em que vários objetivos devem ser alcançados de forma simultânea (GOMES; GONZÁLEZ; CARIGNANO, 2004; ROMERO LÓPEZ, 1993). Esses métodos são aplicados desde a decisão de localização de estádios para grandes eventos, como a Copa do Mundo, à sustentabilidade de produções agrícolas (GOMES JÚNIOR et al., 2011; LACERDA; JÚNIOR, 2013).

Existem vários métodos que podem ser aplicados na tomada de decisão multicritério, entre eles, o Prometee, o MACBETH, o VIP Analysis e o AHP (BANA E 
COSTA; VANSNICK, 1994; BRANS; MARESCHAL, 2005; ZAHEDI, 1986). Para esse estudo, aplicamos o método Analytic Hierarchy Process conhecido pela sigla AHP. Este método foi criado por Saaty (1988) e, segundo o autor, inclui a identificação e a organização dos objetivos da decisão, critérios, restrições e alternativas relevantes. Após essa seleção, são avaliados e estes são hierarquizados e avaliados comparativamente de forma pareada e, por fim, o algoritmo é utilizado para a síntese dos resultados apontando resultados (SAATY, 1988).

Kurttila et al. (2000) afirma que, para a solução do algoritmo criado por Saaty, é necessária a criação da matriz $A$, onde $a_{i j}$ é a comparação dos critérios i e j; $z_{i}$ é a importância relativa do item e tem sua amplitude de 1 a 9 (que pode ser atribuída de acordo com a Tabela 1).

Tabela 1 - Escala Fundamental de Saaty

Valor

Relativa

Recomendação do uso

\section{importância}

1 Igual importância

As duas atividades contribuem igualmente para o objetivo.

3 Importância pequena de A experiência e o juízo favorecem uma atividade em uma sobre outra relação à outra.

5 Importância grande ou A experiência ou juízo favorece fortemente uma essencial atividade em relação à outra.

7 Importância muito grande Uma atividade é muito fortemente favorecida em ou demonstrada relação à outra. Pode ser demonstrada na prática.

9 Importância absoluta A evidência favorece uma atividade em relação à outra, com o mais alto grau de segurança.

2, 4, 6,8 Valores intermediários Quando se procura uma condição de compromisso entre duas definições.

Fonte: Adaptado de Gomes et al. (2004) 
Se a frações $z_{1} / z_{2}=1 / 1$ representam que os critérios $z_{1}$ e $z_{2}$ têm igual importância e 9/1 indica que $z_{1}$ tem extrema importância sobre $z_{2}$ :

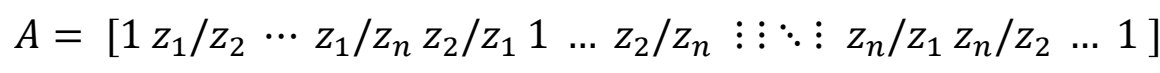

A matriz A de comparação possui ordem nxn, onde n é a quantidade de critérios estabelecidos para a tomada de decisão. No entanto, as comparações na matriz A podem apresentar inconsistências. Para solucionar esses problemas utiliza-se a técnica do eigenvalue, conforme a equação abaixo:

$$
\left(A-\lambda_{\max } I\right) q=0
$$

Onde $\lambda_{\text {max }}$ é o maior eigenfactor da matriz A, I é uma matriz identidade e q é o correto eigenfactor. A variável q constitui, assim, a estimativa das prioridades relativas. Se o tomador de decisão não apresenta inconsistências, q é a estimativa exata do vetor de prioridades. O método AHP mostra que a matriz recíproca de A é sempre maior ou igual a n. Assim, se o pareamento entre os critérios não apresenta quaisquer inconsistência, $\lambda_{\max }=\mathrm{n}$. Baseando-se nessa equação, Saaty (1988) criou um índice de inconsistência $\mathrm{C}_{\mathrm{i}}$ que pode ser calculado com a seguinte fórmula:

$$
C_{i}=\frac{\left(\lambda_{\max }-n\right)}{(n-1)}
$$

Onde $C_{i}$ representa o nível de consistência a respeito da matriz de comparação. Como $C_{i}$ é dependente da quantidade de fatores, uma taxa de consistência $T_{C}$ controla essa dependência. Para calcular essa taxa, é gerada uma matriz média de consistência com dados randomizados $A R$ que varia conforme o tamanho da matriz. Assim $T_{c}$ pode ser obtido da seguinte forma:

$$
T_{c}=100 \frac{C_{i}}{A R}
$$


Por convenção, são considerados aceitáveis valores de $T_{c}$ abaixo de 0,10 . Caso o valor desta taxa esteja acima desse marco, algumas comparações devem ser repetidas até que a taxa de consistência se encontre abaixo de $10 \%$.

\section{Metodologia}

O presente estudo tem uma abordagem quantitativa quanto aos meios, e descritiva quanto aos fins. O universo pesquisado refere-se aos estudantes e ex-estudantes do Programa de Pós-graduação em Extensão Rural da Universidade Federal de Viçosa. O grupo foi escolhido por sua notoriedade no meio acadêmico sobre o conhecimento do tema.

Os dados foram coletados através de formulário na plataforma web, disponível no Apêndice 1, enviado por correio eletrônico a todos estudantes e ex-estudantes que tinham seu endereço eletrônico atualizado na secretaria do Programa, totalizando 45 estudantes. Foram obtidas nove respostas completas, ou seja, que não deixaram questões em branco. Apesar do baixo número de respondentes, a metodologia AHP pode ser aplicada a esse total.

Quadro 1 - Síntese das características constitutivas do perfil relativo ao "Modo de vida rural" e ao "Modo de vida Urbano"

\begin{tabular}{l|l|l}
\hline MODOS DE VIDA / & MODO DE VIDA RURAL & MODO DE VIDA URBANO \\
CARACTERÍSTICAS & & \\
\hline $\mathbf{1}$ ESTILO DE VIDA & & \\
\hline $\mathbf{1 . 1}$ Padrões de & & $\begin{array}{l}\text { Gastos com alimentação } \\
\text { (alimentos comprados e locais } \\
\text { consumo }\end{array}$ \\
\hline 1.1 .1 Alimentação & Presença significativa do autontação). \\
\hline
\end{tabular}




\begin{tabular}{|c|c|c|}
\hline 1.1.2 Vestuário & $\begin{array}{l}\text { Gasto restrito com vestuário } \\
\text { (ocasiões especiais). }\end{array}$ & Gastos casuais com vestuário. \\
\hline 1.1.3 Lazer & Gasto restrito com lazer. & Gastos casuais com lazer. \\
\hline \multicolumn{3}{|l|}{ 1.2 Modos de morar } \\
\hline 1.2.1 Móveis & $\begin{array}{l}\text { Predomínio de mobília que passa } \\
\text { de geração para geração. }\end{array}$ & $\begin{array}{l}\text { Predomínio de mobília } \\
\text { adquirida anualmente. }\end{array}$ \\
\hline 1.2.2 Eletrodomésticos & Básicos. & Diversificados. \\
\hline $\begin{array}{l}\text { 1.2.3 Meios de } \\
\text { comunicação }\end{array}$ & Básicos. & Diversificados. \\
\hline $\begin{array}{l}\text { 1.2.4 Meios de } \\
\text { transporte }\end{array}$ & Coletivo. & Próprio. \\
\hline $\begin{array}{l}\text { 1.2.5 Estrutura física da } \\
\text { casa (número de } \\
\text { cômodos, existência ou } \\
\text { não de saneamento } \\
\text { básico, de eletricidade) }\end{array}$ & Pouco alterada ao longo dos anos. & $\begin{array}{l}\text { Alterada com constância ao } \\
\text { longo dos anos. }\end{array}$ \\
\hline \multicolumn{3}{|l|}{1.3 Tempo gasto } \\
\hline 1.3.1 No trabalho & $\begin{array}{l}\text { Sem demarcações para descanso e } \\
\text { lazer. }\end{array}$ & $\begin{array}{l}\text { Com demarcações para } \\
\text { descanso e lazer. }\end{array}$ \\
\hline 1.3.2 Para o lazer & Pequena importância. & Grande importância. \\
\hline \multicolumn{3}{|l|}{$\begin{array}{l}\text { 1.4 Práticas culturais e } \\
\text { de lazer }\end{array}$} \\
\hline 1.4.1 Práticas religiosas & Muito expressiva. & Secundárias. \\
\hline
\end{tabular}




\begin{tabular}{|c|c|c|}
\hline 1.4.2 Práticas esportivas & Masculinas. & Masculinas e femininas. \\
\hline 1.4.3 Atividades festivas & Em datas tradicionais. & $\begin{array}{l}\text { Casuais e com a presença de } \\
\text { jovens e idosos. }\end{array}$ \\
\hline $\begin{array}{lll}\text { 1.4.4 } & \text { Locais } & \text { para } \\
\text { diversão } & \text { (bares, clubes } \\
\text { etc.) } & & \end{array}$ & Sem especificidade geracional. & Com especificidade geracional. \\
\hline $\begin{array}{l}\text { 1.4.5 Outras atividades } \\
\text { de lazer }\end{array}$ & Inexpressivas. & Diversificadas. \\
\hline $\begin{array}{l}\text { 1.5 Práticas políticas e } \\
\text { associativas }\end{array}$ & & \\
\hline 1.5.1 Sindicato & Masculino. & Presença feminina constante. \\
\hline 1.5.2 Partido & Masculino. & Presença feminina constante. \\
\hline $\begin{array}{l}\text { 1.5.3 Movimentos } \\
\text { sociais }\end{array}$ & Masculino. & Presença feminina constante. \\
\hline $\begin{array}{l}\text { 1.5.4 Associação/ } \\
\text { Cooperativa }\end{array}$ & Masculino. & Presença feminina constante. \\
\hline 1.6 Capital cultural & & \\
\hline 1.6.1 Anos de estudo & Não passa de quatro anos. & Acima de seis anos. \\
\hline $\begin{array}{l}\text { 1.6.2 Qualificação } \\
\text { profissional }\end{array}$ & Ausente. & Presente. \\
\hline 1.7 Capital econômico & & \\
\hline 1.7.1 Renda & Predominantemente agrícola. & $\begin{array}{l}\text { Predominantemente } \\
\text { agrícola. }\end{array}$ \\
\hline
\end{tabular}




\begin{tabular}{|c|c|c|}
\hline $\begin{array}{l}\text { 1.7.2 Pensão, } \\
\text { aposentadoria, }\end{array}$ & Utilizada nas atividades agrícolas. & $\begin{array}{l}\text { Utilizada nas atividades não } \\
\text { agrícolas. }\end{array}$ \\
\hline $\begin{array}{l}\text { 1.7.3 Auxílio do } \\
\text { Governo }\end{array}$ & Acessado por homens. & $\begin{array}{l}\text { Acessado constantemente por } \\
\text { mulheres. }\end{array}$ \\
\hline $\begin{array}{l}\text { 1.8 Frequência de } \\
\text { deslocamento para a } \\
\text { cidade }\end{array}$ & Esporádica. & Semanal. \\
\hline 2 TRABALHO & & \\
\hline $\begin{array}{l}\text { 2.1 Tipo de vínculo de } \\
\text { trabalho }\end{array}$ & $\begin{array}{l}\text { Sem contrato/sem carteira } \\
\text { assinada. }\end{array}$ & Com contrato/carteira assinada. \\
\hline $\begin{array}{l}2.2 \text { Realização do } \\
\text { trabalho }\end{array}$ & Pela família. & $\begin{array}{l}\text { Com mão de obra contratada } \\
\text { esporádica e permanentemente. }\end{array}$ \\
\hline 2.2.1 Assistência técnica & Ocasional. & Frequente. \\
\hline $\begin{array}{l}\text { 2.3 Forma de gerir a } \\
\text { propriedade }\end{array}$ & Sem utilizar planilha de custo. & Utilizando planilha de custos. \\
\hline $\begin{array}{l}\text { 2.4 Aparato tecnológico } \\
\text { produtivo }\end{array}$ & & \\
\hline 2.4.1 Máquinas & Antigas. & Novas. \\
\hline 2.4.2 Insumos & Pouco usados. & Muito usadas. \\
\hline $\begin{array}{l}\text { 2.4.3 Mudanças nas } \\
\text { práticas produtivas }\end{array}$ & Passadas de pai para filho. & $\begin{array}{l}\text { Adquiridas através de } \\
\text { capacitação profissional. }\end{array}$ \\
\hline 3 Acesso a serviços & & \\
\hline 3.1 Escola (graus) & Ensino Básico e Fundamental. & Ensino Médio e Superior. \\
\hline
\end{tabular}




\begin{tabular}{l|l|l}
\hline $\begin{array}{l}\text { 3.2 Hospitais, postos de } \\
\text { saúde }\end{array}$ & Uso municipal. & Uso regional. \\
\hline $\mathbf{3 . 3 \quad \text { Atendimento }}$ & Inexistente. & \\
jurídico & & Presente. \\
\hline 3.4 Outros & Pouco presentes. & \\
\hline
\end{tabular}

Fonte: Fiúza e Pinto (2012)

Para verificar qual o peso relativo de cada um destes vetores foi empregado o método Analytic Hierarchy Process (AHP). Este método, criado por Saaty (1988), é adequado para esse estudo, pois se trata de uma decisão de alta complexidade e com opções que podem ser estruturadas de forma hierárquica. Apesar de adequado, o método possui limitações, a principal delas é o fato de considerar que há uma estrutura hierárquica para resolução de todos os problemas. Para a análise dos dados, utilizou-se o programa Expert Choice, Versão 11, no qual foram realizados os procedimentos para a realização do Analytic Hierarchy Process (AHP).

\section{Análise dos dados}

A estruturação dos itens apresentados do Quadro 1 seguiram o organograma apresentado na Figura 1, mostrada na sequência do estudo:

Figura 1 - Hierarquia utilizada na realização do AHP

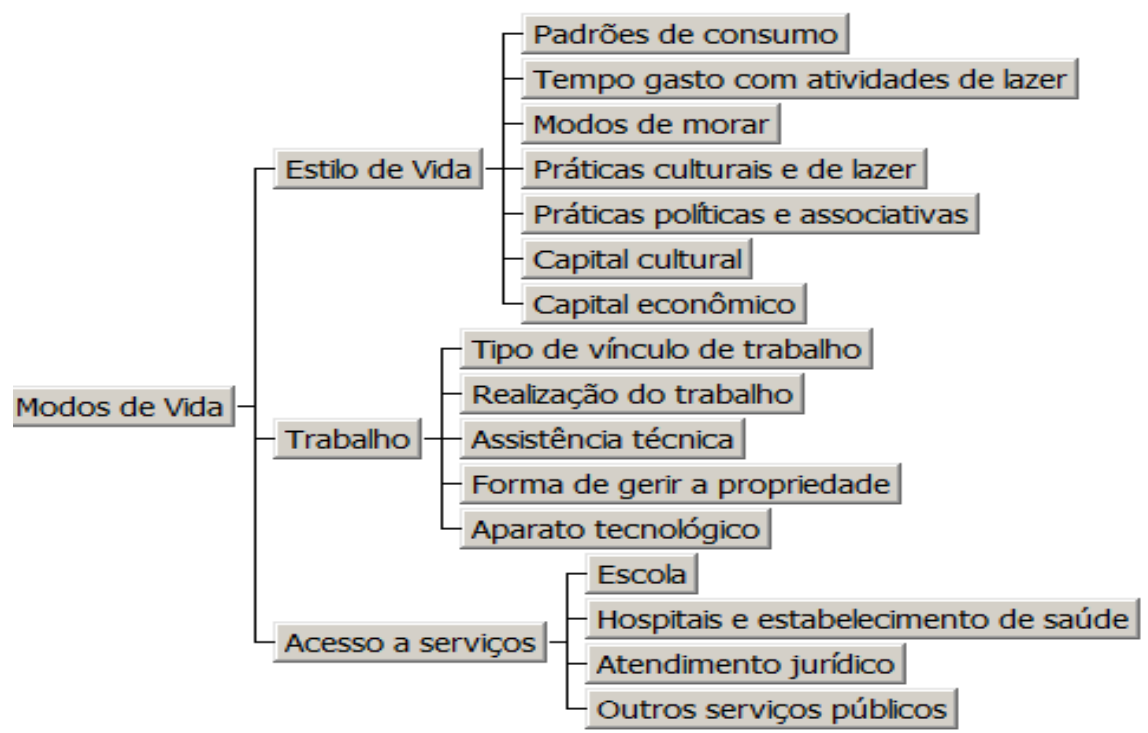

Fonte: Elaborado pelo autor 
Como representado, foram utilizados três níveis hierárquicos onde se procurou definir quais seriam os pesos de cada uma dessas variáveis para a criação de índices de integração à sociedade de mercado. Uma análise prévia das respostas sugere que grande parte das variáveis apresentou todas as respostas favoráveis à inserção das mesmas no índice, com exceção de "Padrões de consumo" e "Práticas e políticas associativas", que obtiveram somente uma resposta considerando que essas não contribuíram com o índice (Apêndice 2).

Os resultados da combinação dos entrevistados pela metodologia AHP aponta para a viabilidade do índice dado às variáveis da Figura 1. A opção das variáveis que contribuem para a definição do modo de vida mostrou-se como a melhor alternativa, e esses resultados não apresentaram inconsistência, conforme a Figura 2. Esses resultados repetem-se mesmo no estudo de cada entrevistado de forma isolada.

Figura 2 - Resultado combinado das alternativas com a aplicação do AHP

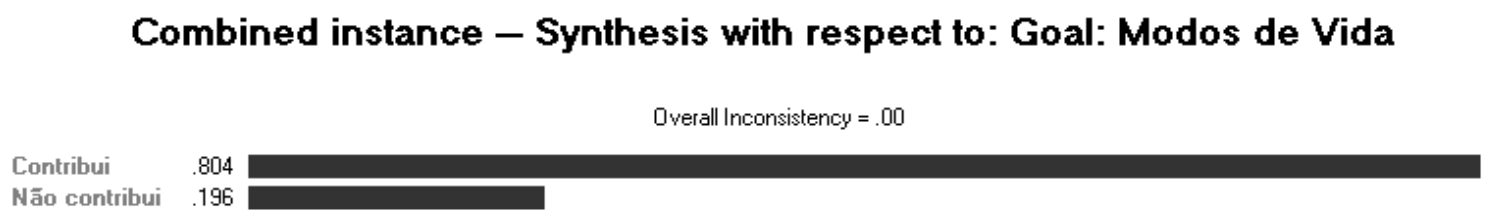

Fonte: Elaborado pelo autor

O resultado favorável às variáveis selecionadas não apresentou sensibilidade às variáveis do segundo nível hierárquico, assim como no terceiro nível. Demonstrou-se assim que, independentemente da alteração dos pesos dados a essas variáveis, a hipótese de que as variáveis selecionadas não contribuíram para o índice proposto não ocorreria em nenhum cenário, o que demonstra uma consonância entre os entrevistados e a obra de Beaujeu-Garnier (1997), Endlich (2010) e, principalmente, de Fiúza e Pinto (2012). 
Figura 3 - Sensibilidade dos nós de segundo nível para a meta "Modo de vida"

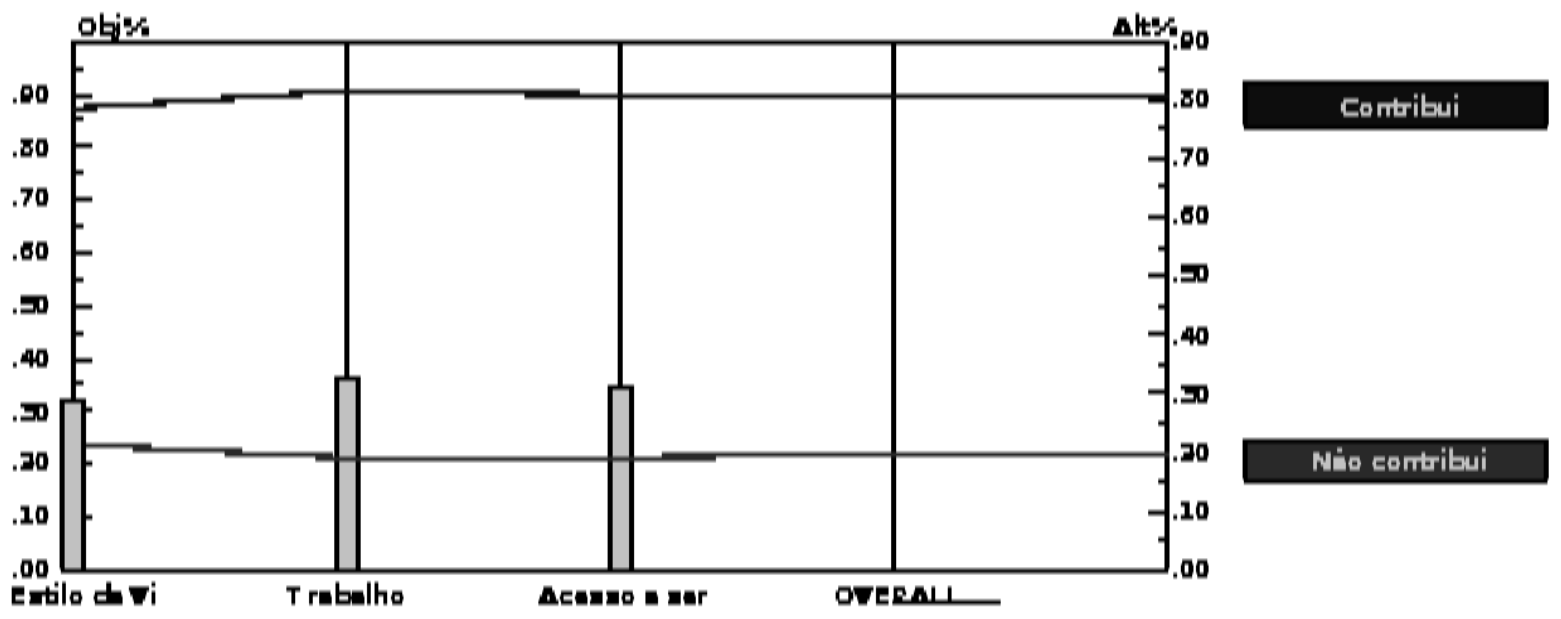

Fonte: Elaborado pelo autor

Os pesos que contribuíram para a criação do índice constam na Tabela 2, na qual também é possível visualizar quanto cada uma delas contribuiu para a escolha das variáveis para a criação do índice. Como visto em todas as variáveis, creditam a hipótese de contribuição para o índice, assim, não apresentam a necessidade de exclusão, mostrando que os entrevistados consideraram possível a criação do índice de integração à sociedade capitalista de mercado possível com as variáveis apontadas por Fiúza e Pinto (2012).

Tabela 2 - Pesos das variáveis e contribuição

\begin{tabular}{|c|c|c|c|c|}
\hline Level 1 & Level 2 & $\begin{array}{l}\text { Contrib } \\
\text { ui }\end{array}$ & $\begin{array}{c}\text { Não } \\
\text { contrib } \\
\text { ui }\end{array}$ & $\begin{array}{l}\text { Total } \\
\text { geral }\end{array}$ \\
\hline \multirow[t]{3}{*}{$\begin{array}{l}\text { Acesso a serviços (L: } \\
\text {,335) }\end{array}$} & Atendimento jurídico (L: ,248) & 0,067 & 0,017 & 0,084 \\
\hline & Escola (L: ,256) & 0,069 & 0,013 & 0,082 \\
\hline & $\begin{array}{l}\text { Hospitais e estabelecimento de } \\
\text { saúde (L: ,256) }\end{array}$ & 0,069 & 0,018 & 0,087 \\
\hline
\end{tabular}




\begin{tabular}{|c|c|c|c|c|}
\hline & Outros serviços públicos (L: ,240) & 0,064 & 0,016 & 0,08 \\
\hline $\begin{array}{l}\text { Acesso a serviços (L: } \\
\text {,335) Total }\end{array}$ & & 0,269 & 0,064 & 0,333 \\
\hline \multirow[t]{8}{*}{ Estilo de vida (L: ,310) } & Capital cultural (L: ,094) & 0,024 & 0,009 & 0,033 \\
\hline & Capital econômico (L: ,137) & 0,034 & 0,006 & 0,04 \\
\hline & $\begin{array}{l}\text { Frequência de deslocamento para a } \\
\text { cidade (L: ,086) }\end{array}$ & 0,021 & 0,005 & 0,026 \\
\hline & Modos de morar (L: ,135) & 0,034 & 0,007 & 0,041 \\
\hline & Padrões de consumo (L: ,157) & 0,039 & 0,01 & 0,049 \\
\hline & Práticas culturais e de lazer (L:, 140$)$ & 0,035 & 0,01 & 0,045 \\
\hline & $\begin{array}{l}\text { Práticas políticas e associativas (L: } \\
\text {,095) }\end{array}$ & 0,024 & 0,01 & 0,034 \\
\hline & $\begin{array}{l}\text { Tempo gasto com atividades de } \\
\text { lazer (L: 156) }\end{array}$ & 0,039 & 0,013 & 0,052 \\
\hline $\begin{array}{l}\text { Estilo de vida }(\mathrm{L}:, 310) \\
\text { Total }\end{array}$ & & 0,25 & 0,07 & 0,32 \\
\hline \multirow[t]{5}{*}{ Trabalho (L: ,355) } & Aparato tecnológico (L: ,189) & 0,054 & 0,01 & 0,064 \\
\hline & Assistência técnica (L: ,184) & 0,053 & 0,011 & 0,064 \\
\hline & $\begin{array}{l}\text { Forma de gerir a propriedade (L: } \\
\text {,211) }\end{array}$ & 0,06 & 0,011 & 0,071 \\
\hline & Realização do trabalho (L: ,224) & 0,064 & 0,022 & 0,086 \\
\hline & Tipo de vínculo de trabalho (L: ,191) & 0,054 & 0,011 & 0,065 \\
\hline Trabalho (L: ,355) Total & & 0,285 & 0,065 & 0,35 \\
\hline Total geral & & 0,804 & 0,199 & 1,003 \\
\hline
\end{tabular}

Fonte: Elaborado pelo autor 
De acordo com os resultados, todas as possíveis variáveis utilizadas seriam de alta importância na composição do índice e corroborando, dessa forma, os estudos de Fiúza e Pinto (2012) que selecionam essas variáveis para a caracterização do modo de vida "rural" e "urbano" e a consequente integração a sociedade capitalista de mercado.

\section{Considerações finais}

A discussão sobre formas de definição de rural e urbano continuam a oferecer grandes embates teóricos. Dentro dessa contenda literária, destaca-se a definição do rural e o urbano como modo de vida. Para solidificar com bases empíricas dessa corrente, a criação de um índice que mensura a integração à sociedade capitalista de mercado é um canal viável, como apresentado nos resultados deste estudo.

A utilização dos proxies apontadas por Fiúza e Pinto (2012) são consideradas como uma contribuição satisfatória entre os entrevistados na pesquisa. Os resultados apontam, ainda, para os pesos de cada variável calculados pela metodologia AHP que possibilitam o cálculo e a aferição de importância relativa das variáveis estudadas. É possível acrescentar, ainda, que todas as variáveis estudadas apresentaram-se viáveis para a estruturação de um índice entre os entrevistados.

Devido às limitações metodológicas e da alta complexidade do tema abordado, ainda sem consensos na literatura. Além da impossibilidade de acesso a mais pesquisadores do tema não foi possível verificar se a estrutura adotada no estudo foi ótima. Por isso, futuros estudos devem procurar meios de contornar essas limitações, bem como de calcular empiricamente, em diversas regiões do globo, o índice proposto a fim de dar continuidade à agenda de pesquisa aqui estabelecida. 


\section{Referências bibliográficas}

ABRAMOVAY, R. Funções e medidas da ruralidade no desenvolvimento contemporâneo. Rio de Janeiro: IPEA, 2000.

BANA E COSTA, C. A.; VANSNICK, J. C. MACBETH: an interactive path towards the construction of cardinal value functions. International transactions in operational research, v. 1, n. 4, p. 489-500, 1994.

BEAUJEU-GARNIER, J. Géographie urbaine. Paris: Armand Colin, 1997.

BERNARDELLI, M. L. F. da H. O caráter urbano das pequenas cidades da região de CatanduvaSP. In: SPOSITO, M. E. B.; WHITACKER, A. M. (eds.). Cidade e campo: relações e contradições entre urbano e rural. São Paulo: Expressão Popular, 2010.

BRAGA, G. B.; REMOALDO, P. C.; FIÚZA, A. L. DE C. A methodology for definition of rural spaces: an implementation in Brazil. Ciência Rural, v. 46, n. 2, p. 375-380, fev. 2016.

BRANS, J. P.; MARESCHAL, B. PROMETHEE methods. In: Multiple criteria decision analysis: state of the art surveys. [s.1.]: Springer, 2005, p. 163-186.

ENDLICH, Â. M. Perspectivas sobre o urbano e o rural. In: SPOSITO, M. E. B.; WHITACKER, A. M. (eds.). Cidade e campo: relações e contradições entre urbano e rural. São Paulo: Expressão Popular, 2010, p. 11-31.

FIÚZA, A. L. de C.; PINTO, N. M. de A. The contribuition of conceptual distinciction between "field" and "rural" and "city" and "urban" for researchers of rural sociology. XIII World Congress of Rural Sociology. July 29 to August 4. Anais... Lisboa: 2012.

GOMES JÚNIOR, S. F. et al. Integração de métodos multicritério na busca da sustentabilidade agrícola para a produção de tomates no município de São José de Ubá-RJ. Pesquisa Operacional, v. 31 , n. 1 , p. $157-171,2011$.

GOMES, L. F. A. M.; GONZÁleZ, M. C. A.; CARIGNANO, C. Tomada de decisões em cenários complexos: introdução aos métodos discretos do apoio multicritério à decisão. São Paulo: Thomson, 2004.

KAGEYAMA, Â. Desenvolvimento rural: conceitos e aplicação ao caso brasileiro. Porto Alegre: Editora da UFRGS, 2008.

KURTTILA, M. et al. Utilizing the analytic hierarchy process (AHP) in SWOT analysis: a hybrid method and its application to a forest-certification case. Forest Policy and Economics, v. 1, n. 1, p. 41-52, 1/maio/2000.

LACERDA, L. S.; JÚNIOR, S. F. G. Um estudo multicritério para a escolha das cidades-sede para a Copa do Mundo FIFA 2014. Pesquisa Operacional para o Desenvolvimento, v. 5, n. 1, p. 100-112, 2013.

ROMERO LÓPEZ, C. Teoría de la decisión multicriterio: conceptos, técnicas y aplicaciones. [s.1.]: Alianza Editorial, 1993. 
RYBCZYNSKI, W. A vida nas cidades: expectativas urbanas no novo mundo. Rio de Janeiro: Record, 1996.

SAATY, T. L. What is the analytic hierarchy process? [s.l.]: Springer, 1988.

SANCHEZ-CASAS, C. La centralidad de el modo de assentamiento durante el predominio de la contradicción campo-cidade. In: Sociedad, sistema y habitat. Madri: Los libros de la Catarata, 2009.

VEIGA, J. E. da. Cidades imaginárias: o Brasil é menos urbano do que se calcula. São Paulo: Autores Associados, 2002.

(2010)

ZAHEDI, F. The analytic hierarchy process: a survey of the method and its applications. interfaces, v. 16, n. 4, p. 96-108, 1986. 